\title{
Correction to: Confirmatory factor analyses of the ORTO 15-, 11- and 9-item scales and recommendations for suggested cut-off scores
}

\author{
Stephan Moller ${ }^{1} \cdot$ Pragalathan Apputhurai $^{1} \cdot$ Simon R. Knowles ${ }^{1,2,3,4,5}$
}

Published online: 1 June 2019

(c) Springer Nature Switzerland AG 2019

\section{Correction to: \\ Eating and Weight Disorders - Studies on Anorexia, \\ Bulimia and Obesity (2019) 24:21-28 \\ https://doi.org/10.1007/s40519-018-0515-0}

Due to an unfortunate error, interpretation of the ORTO-7 cut-off score is incorrect.

The errors in the manuscript are located in three places:

1. Last sentence of the Abstract.

"Analysis of cut-offs revealed that a cut-off score of equal or greater than 19 on the ORTO-7 represents probable orthorexia." should read "Analysis of cut-offs revealed that a cut-off score of equal or less than 19 on the ORTO-7 represents probable orthorexia.".

2. In the Discussion section (fifth sentence in the third paragraph).

"Based on an ROC analyses, scores equal to 19 or more are likely to represent probable orthorexia." should read "Based on an ROC analyses, scores equal to 19 or less are likely to represent probable orthorexia.".

3. In the Discussion section (Third sentence in the last paragraph).

The original article can be found online at https://doi.org/10.1007/ s40519-018-0515-0.

Simon R. Knowles

sknowles@swin.edu.au

1 Faculty of Health Arts, and Design, Swinburne University of Technology, Melbourne, Australia

2 Department of Gastroenterology, Royal Melbourne Hospital, Melbourne, Australia

3 Department of Gastroenterology, The Alfred Hospital, Melbourne, Australia

4 Department of Mental Health, St Vincent's Hospital, Melbourne, Australia

5 University of Melbourne, Melbourne, Australia
"A cut-off score equal to 19 or more is likely to represent probable orthorexia." should read "A cut-off score equal to 19 or less is likely to represent probable orthorexia.".

We apologize for the errors made.

Publisher's Note Springer Nature remains neutral with regard to jurisdictional claims in published maps and institutional affiliations. 